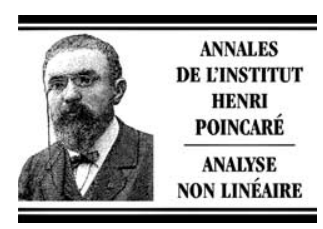

www.elsevier.com/locate/anihpc

\title{
On planar selfdual Electroweak vortices
}

\section{Sur les vortex Électrofaibles autoduaux dans le plan}

\author{
Dongho Chae ${ }^{\mathrm{a}}$, Gabriella Tarantello ${ }^{\mathrm{b}, *}$ \\ a Department of Mathematics, Seoul National University, Seoul 151-742, South Korea \\ b Dipartimento di Matematica, Università degli Studi di Roma "Tor Vergata”, Via della Ricerca Scientifica, O0133 Roma, Italy \\ Received 29 May 2002; accepted 30 January 2003
}

\begin{abstract}
By perturbation techniques, we obtain a new class of selfdual Electroweak vortices in $\mathbb{R}^{2}$, whose asymptotic behavior we control in an "optimal" way to yield a sort of "quantization" property for the corresponding flux.

Our class of vortex-solutions complements that constructed by Spruck and Yang [Comm. Math. Phys. 144 (1992) 215-234].

(C) 2004 L'Association Publications de l'Institut Henri Poincaré. Published by Elsevier B.V. All rights reserved
\end{abstract}

\section{Résumé}

En utilisant des techniques de perturbation, nous obtenons une nouvelle classe de vortex Électrofaibles autoduaux dans $\mathbb{R}^{2}$, dont nous pouvons contrôler de façon «optimale» le comportement asymptotique en donnant une propriété de «quantisation» pour le flot correspondant.

Notre classe de solutions complète celles construites par Spruck et Yang [Comm. Math. Phys. 144 (1992) 215-234].

(C) 2004 L'Association Publications de l'Institut Henri Poincaré. Published by Elsevier B.V. All rights reserved

MSC: 35J45; 35J60; 37K40; 70S15

\section{Introduction}

We shall be concerned with planar vortex-type (self-dual) solutions for the celebrated $S U(2) \times U(1)$ Electroweak theory of Glashow, Salam and Weinberg [10]. As observed by Ambjorn and Olesen [2-4], if the physical parameters satisfy a suitable critical condition, it is possible to determine Bogomol'nyi type equations (also known as self-dual equations) to be satisfied by Electroweak-vortices when expressed in terms of the unitary gauge variables. The selfdual equations include a gauge invariant version of the Cauchy-Riemann equation, which makes it reasonable to assume that the $W$-boson field admits a finite number $N$ of zeroes (counted with

\footnotetext{
* Corresponding author.

E-mail addresses: dhchae@math.snu.ac.kr (D. Chae), tarantel@mat.uniroma2.it (G. Tarantello).
} 
multiplicity). Such an integer $N$ is called the vortex number, and the corresponding vanishing points of W are called the vortex points.

Moreover, by means of an approach introduced by Taubes $[15,16]$ in the context of Ginzburg-Landau vortices, it is possible to reduce the analysis of self-dual Electroweak vortices to the study of the following elliptic system:

$$
\left\{\begin{array}{l}
-\Delta u_{1}=4 g^{2} \mathrm{e}^{u_{1}}+g^{2} \mathrm{e}^{u_{2}}-4 \pi \sum_{k=1}^{m} n_{k} \delta\left(z-z_{k}\right), \\
\Delta u_{2}=\frac{g^{2}}{2 \cos ^{2} \theta}\left(\mathrm{e}^{u_{2}}-\phi_{0}^{2}\right)+2 g^{2} \mathrm{e}^{u_{1}}
\end{array}\right.
$$

where $\phi_{0}$ is a given positive parameter, $g$ is the $S U(2)$-coupling constant, and $\theta \in(0, \pi / 2)$ is the so called "Weinberg-angle", related with the $U(1)$-coupling constant $g_{*}$ via the relation,

$$
\cos \theta=\frac{g}{\left(g^{2}+g_{*}^{2}\right)^{1 / 2}}
$$

We refer to the recent monograph of Y. Yang [17] and [5] for details, and, in particular, on how to recover the full vortex configuration out of solutions of $(\mathcal{P})$. We only mention that, by virtue of the vortex ansatz and accordingly to the unitary gauge variables, the vortex solution is specified by the (complex valued) massive field $W$, the (scalar) field $\varphi$ and the real valued 2-vector fields $P=\left(P_{\mu}\right)_{\mu=1,2}$ and $Z=\left(Z_{\mu}\right)_{\mu=1,2}$. So that, $u_{1}$ and $u_{2}$ determine the magnitude of $W$ and $\varphi$ respectively, as follows:

$$
\varphi^{2}=\mathrm{e}^{u_{2}}, \quad|W|^{2}=\mathrm{e}^{u_{1}}
$$

Thus, $z_{k}$ corresponds to a vortex point, the integer $n_{k} \in \mathbb{N}$ to its multiplicity $k=1, \ldots, m$, and $N=\sum_{k=1}^{m} n_{k}$ is the vortex number.

Furthermore, while the 2-vector fields $P$ and $Z$ are defined only up to gauge transformations, their gauge invariant curls,

$$
P_{12}=\partial_{1} P_{2}-\partial_{2} P_{1} \quad \text { and } \quad Z_{12}=\partial_{1} Z_{2}-\partial_{2} Z_{1}
$$

are determined by the relations:

$$
\begin{aligned}
& P_{12}=\frac{g}{2 \sin \theta} \phi_{0}^{2}+2 g \sin \theta|W|^{2}, \\
& Z_{12}=\frac{g}{2 \cos \theta}\left(\varphi^{2}-\phi_{0}^{2}\right)+2 g \cos \theta|W|^{2}
\end{aligned}
$$

(see [17]). Following the numerical evidence provided by Ambjorn and Olesen, the first rigorous results concerning Electroweak-vortices have been obtained by Spruck and Yang in [13,14]. In [13], the authors aim to obtain configurations of the type described in physics literature as the "Abrikosov's mixed states" (see [1]), so they consider the selfdual equations subject to 't Hooft boundary conditions [9]. In turn, this amounts to solve $(\mathcal{P})$ under periodic boundary conditions, and Spruck and Yang in [13] obtain necessary and sufficient conditions on the given physical parameters, in order to ensure the presence of periodic $N$-vortices in the theory. Their results are sharp for $N=1,2$ and recently have been improved by Bartolucci and Tarantello in [5] to a wider range of parameters.

In this paper, we shall be concerned with planar vortex-type configurations, and consider $(\mathcal{P})$ over $\mathbb{R}^{2}$ subject to appropriate decay assumptions at infinity. This situation has been considered in [14], where it has been observed that necessarily, the corresponding vortex-solutions must carry infinite energy. As a consequence, solutions of $(\mathcal{P})$ over $\mathbb{R}^{2}$ appear in abundance, and are distinguished according to their rate of decay at infinity.

In view of (0.1), also the flux of the fields $P$ and $Z$ is infinite, while this may not be necessarily the case for the field: $(\sin \theta) P+(\cos \theta) Z$, which bares informations about the gauge potential in the original variables. We shall be concerned with such "finite-flux" selfdual solutions, by solving $(\mathcal{P})$ under the condition:

$$
\mathrm{e}^{u_{1}}, \mathrm{e}^{u_{2}} \in L^{1}\left(\mathbb{R}^{2}\right)
$$


In fact, by setting:

$$
\sigma_{1}=\frac{2 g^{2}}{\pi} \int_{\mathbb{R}^{2}} \mathrm{e}^{u_{1}}, \quad \sigma_{2}=\frac{g^{2}}{2 \pi} \int_{\mathbb{R}^{2}} \mathrm{e}^{u_{2}}
$$

in view of $(0.1),(0.2)$ we see that the flux $\Phi$ of the field $(\sin \theta) P+(\cos \theta) Z$ is given by,

$$
\Phi=\int_{\mathbb{R}^{2}}\left(\sin \theta P_{12}+\cos \theta Z_{12}\right)=\frac{\pi}{g}\left(\sigma_{1}+\sigma_{2}\right) .
$$

As a first fact, we show that necessarily

$$
\sigma_{1}+\sigma_{2}>2(N+1)
$$

as $\sigma_{1}$ and $\sigma_{2}$ determine the rate of decay at infinity of $u_{1}$ and $u_{2}$ (see Theorem 1.1).

The existence results for $(\mathcal{P})-(0.3)$ derived by Spruck and Yang in [14] imply that the lower bound (0.6) is "sharp", as they construct solutions satisfying:

$\sigma_{2}>2 N ; \quad \sigma_{1}+\sigma_{2}$ any assigned value in $(2(N+1),+\infty)$.

Notice that, in contrast to the periodic case, this class of solutions lacks any sort of "quantization" property for the corresponding flux (0.5). A first goal of this paper is to show that, actually, this is no longer the case, if we consider solutions of $(\mathcal{P})-(0.3)$ with $\sigma_{2} \leqslant 2 N$.

In this case, and when all vortex points coincide, we show that the value $\sigma_{2}$ also controls from above the value $\sigma_{1}$. This implies that, in certain regime, (e.g., $\sigma_{2}$ small) the lower bound $2(N+1)$ in $(0.6)$ is no longer sharp, as $\sigma_{1}+\sigma_{2}$ is now forced to remain close to the value $4(N+1)$, and any other value is ruled out. See Lemma 1.6.

Thus, for $\sigma_{2}$ small, a sort of "quantization" property is restored as $\sigma_{1}$ "approximately" equals to $4(N+1)$.

Our second goal, is to show that, in fact, such situation does occur. So, in Section 2, we shall focus our attention in constructing several families of solutions of $(\mathcal{P})-(0.3)$ so that

$$
\sigma_{2}=\mathrm{o}(1) \text { and } \sigma_{1}=4(N+1)+\mathrm{o}(1) .
$$

To this end, we provide a priori estimates (see Theorem 1.1), so that the $L^{1}$-norm of $\mathrm{e}^{u_{2}}$ uniformly estimates its $L^{\infty}$-norm. Thus, requiring a small $\sigma_{2}$, implies that we must have $\mathrm{e}^{u_{2}}$ also small in $L^{\infty}$-norm. Therefore, the first equation in $(\mathcal{P})$ may be viewed as a "perturbation" of the following singular Liouville equation:

$$
\left\{\begin{array}{l}
-\Delta u=4 g^{2} \mathrm{e}^{u}-4 \pi \sum_{k=1}^{m} n_{k} \delta\left(z-z_{k}\right) \text { in } \mathbb{R}^{2}, \\
\int_{\mathbb{R}^{2}} \mathrm{e}^{u}<+\infty,
\end{array}\right.
$$

whose solutions may be classified according to Liouville formula (e.g., see [12]). When all vortex points coincide, we are able to adapt a perturbation approach introduced by Chae and Imanuvilov in [7] to construct "nontopological" Chern-Simons vortices, and obtain solutions of $(\mathcal{P})$, "bifurcating" out of solutions of the singular Liouville equation mentioned above. In this way, we are able to provide rather accurate pointwise estimates on our solutions to ensure (0.7), together with a sharp control on their decay rate at infinity. We refer to Theorem 2.1 and 2.2 for the precise statements. Clearly, our result furnishes a new class of solutions for $(\mathcal{P})-(0.3)$ which complements those constructed in [14]. 


\section{Asymptotic behavior}

Consider the problem,

$$
(P) \quad\left\{\begin{array}{l}
-\Delta u_{1}=4 g^{2} \mathrm{e}^{u_{1}}+g^{2} \mathrm{e}^{u_{2}}-4 \pi \sum_{k=1}^{m} n_{k} \delta\left(z-z_{k}\right), \\
\Delta u_{2}=\frac{g^{2}}{2 \cos ^{2} \theta}\left(\mathrm{e}^{u_{2}}-\phi_{0}^{2}\right)+2 g^{2} \mathrm{e}^{u_{1}} \quad \text { in } \mathbb{R}^{2}, \\
\int_{\mathbb{R}^{2}} \mathrm{e}^{u_{1}}<+\infty, \quad \int_{\mathbb{R}^{2}} \mathrm{e}^{u_{2}}<+\infty,
\end{array}\right.
$$

where $\left\{z_{1}, \ldots, z_{m}\right\} \subset \mathbb{R}^{2}$ are given points, $\left\{n_{1}, \ldots, n_{m}\right\} \subset \mathbb{N}$ are given integers, and $\delta(z-p)$ denotes the Dirac measure with pole at $p \in \mathbb{R}^{2}$. Let

$$
N=\sum_{k=1}^{m} n_{k}, \quad c_{0}=\frac{g^{2} \phi_{0}^{2}}{8 \cos ^{2} \theta}
$$

with $g, \phi_{0}$ given positive constants and $\theta \in(0, \pi / 2)$. Set

$$
\sigma_{1}=\frac{2 g^{2}}{\pi} \int_{\mathbb{R}^{2}} \mathrm{e}^{u_{1}}, \quad \sigma_{2}=\frac{g^{2}}{2 \pi} \int_{\mathbb{R}^{2}} \mathrm{e}^{u_{2}} .
$$

We devote this section to obtain the asymptotic behavior, as $|x| \rightarrow+\infty$, of a solution pair $\left(u_{1}, u_{2}\right)$ for $(P)$ in terms of $\sigma_{1}$ and $\sigma_{2}$, and to establish some interesting relation for such values. We have:

Theorem 1.1. Let $\left(u_{1}, u_{2}\right)$ be a solution pair for $(P)$, then

(i) $u_{1}^{+} \in L^{\infty}\left(\mathbb{R}^{2}\right)$,

$$
u_{1}(x) \geqslant\left(2 N-\left(\sigma_{1}+\sigma_{2}\right)\right) \ln |x|-C, \quad \text { as }|x| \rightarrow+\infty
$$

for suitable $C>0$,

$$
\frac{u_{1}(x)}{\ln |x|} \rightarrow 2 N-\left(\sigma_{1}+\sigma_{2}\right), \quad \text { as }|x| \rightarrow+\infty,
$$

and so necessarily

$$
\sigma_{1}+\sigma_{2}>2(N+1) \text {. }
$$

(ii) $u_{2}^{+} \in L^{\infty}\left(\mathbb{R}^{2}\right)$ and for suitable $C_{0}>0$ (depending on $c_{0}$ only)

$$
\left\|\mathrm{e}^{u_{2}}\right\|_{L^{\infty}\left(\mathbb{R}^{2}\right)} \leqslant C_{0}\left\|\mathrm{e}^{u_{2}}\right\|_{L^{1}\left(\mathbb{R}^{2}\right)} .
$$

In addition, if

$$
\frac{u_{2}(x)+c_{0}\left|x-x_{0}\right|^{2}}{1+|x|^{1+\alpha / 2}} \in L^{2}\left(\mathbb{R}^{2}\right)
$$

for some $x_{0} \in \mathbb{R}^{2}$ and $\alpha \in(0,1)$, then

$$
\begin{gathered}
u_{1}(x)=\left(2 N-\left(\sigma_{1}+\sigma_{2}\right)\right) \ln |x|+\mathrm{O}(1) \\
u_{2}(x)=-c_{0}\left|x-x_{0}\right|^{2}+\frac{1}{2}\left(\sigma_{1}+\frac{\sigma_{2}}{\cos ^{2} \theta}\right) \ln |x|+\mathrm{O}(1) \\
\text { as }|x| \rightarrow+\infty
\end{gathered}
$$


In order to derive Theorem 1.1 we are going to recall some basically well known facts about the function:

$$
w(x)=\frac{1}{2 \pi} \int_{\mathbb{R}^{2}}(\ln |x-y|-\ln (1+|y|)) f(y) \mathrm{d} y
$$

with $f \in L^{1}\left(\mathbb{R}^{2}\right)$.

Lemma 1.1. Let

$$
f\left((\ln |f|)^{+}+1\right) \in L^{1}\left(\mathbb{R}^{2}\right) \quad \text { and } \quad \beta=\frac{1}{2 \pi} \int_{\mathbb{R}^{2}} f(y) \mathrm{d} y
$$

(i) if $f \geqslant 0$, then

$$
w(x) \leqslant \beta \ln (|x|+1)+C
$$

for suitable $C>0$;

(ii) $\frac{w(x)}{\ln |x|} \rightarrow \beta, \quad$ as $|x| \rightarrow+\infty$;

(iii) if

$$
\ln (1+|x|) f \in L^{1}\left(\mathbb{R}^{2}\right),
$$

then

$$
w(x)=\beta \ln |x|+\mathrm{O}(1), \quad \text { as }|x| \rightarrow+\infty .
$$

We notice that properties (1.11), (1.12) and (1.14) remain valid for any other solution $v$ of the equation:

$$
\Delta v=f
$$

provided it admits a "slow" growth at infinity as expressed by one of the following conditions:

$$
\frac{v^{+}}{\ln |x|+1} \in L^{\infty}\left(\mathbb{R}^{2}\right)
$$

or

$$
\frac{v}{1+|x|^{1+\alpha / 2}} \in L^{2}\left(\mathbb{R}^{2}\right), \quad \text { for some } \alpha \in(0,1) .
$$

Corollary 1.1. Assume (1.10), and let $v \in L_{\text {loc }}^{1}\left(\mathbb{R}^{2}\right)$ be a solution of (1.15) which satisfies (1.16) or (1.17). Then, properties (i), (ii) and (iii) of Lemma 1.2 hold for $v$.

Proof of Corollary 1.1. Notice that the function $u=v-w$ is harmonic over $\mathbb{R}^{2}$ and, in view of Lemma 1.2, satisfies (1.16) or (1.17). In either case $u$ must be constant. Indeed, this is a well known fact in case (1.16) holds (e.g., see Lemma 4.6.1 in [17]), while it is a consequence of Proposition 1.1 in [7] in case $u$ satisfies (1.17).

Proof of Lemma 1.1. Let us start by observing that

$$
\ln |x-y|-\ln (1+|y|) \leqslant \ln (|x|+1), \quad \forall x, y \in \mathbb{R}^{2} .
$$

Consequently, for $f \geqslant 0$, property (i) can be easily derived. To obtain (ii), let $|x|>1$ and consider,

$$
\sigma(x)=\frac{1}{\ln |x|} \int_{\mathbb{R}^{2}}(\ln |x-y|-\ln (1+|y|)-\ln |x|) f(y) \mathrm{d} y .
$$


Thus, we must show that $\sigma(x) \rightarrow 0$ as $|x| \rightarrow+\infty$. For this purpose, write

$$
\sigma(x)=\sigma_{1}(x)+\sigma_{2}(x)+\sigma_{3}(x),
$$

where $\sigma_{1}, \sigma_{2}$ and $\sigma_{3}$ are defined by taking the integral in (1.18) over the regions $D_{1}=\left\{y \in \mathbb{R}^{2}:|x-y|<1\right\}$, $D_{2}=\left\{y \in \mathbb{R}^{2}:|x-y|>1\right.$ and $\left.|y|<\rho\right\}$ and $D_{3}=\left\{y \in \mathbb{R}^{2}:|x-y|>1\right.$ and $\left.|y|>\rho\right\}$ respectively, with $\rho>1$ a fixed constant. We estimate,

$$
\begin{aligned}
\left|\sigma_{1}(x)\right| \leqslant & \frac{1}{\ln |x|} \int_{\{|x-y|<1\}}|\ln | x-y|-\ln (1+|y|)-\ln | x|||f(y)| \mathrm{d} y \\
\leqslant & \frac{1}{\ln |x|} \int_{\{|x-y|<1\}} \ln \left(\frac{1}{|x-y|}\right)|f(y)| \mathrm{d} y+\frac{\ln (|x|+2)+\ln |x|}{\ln |x|} \int_{\{|x-y|<1\}}|f(y)| \mathrm{d} y \\
\leqslant & \frac{1}{\ln |x|}\left(\int_{\{|x-y|<1\}} \frac{\mathrm{d} y}{|x-y|}+\int_{\{|x-y|<1\}}|f(y)|(\ln |f(y)|)^{+} \mathrm{d} y\right) \\
& +2\left(1+\frac{1}{\ln |x|}\right) \int_{\{|y|>|x|-1\}}|f(y)| \mathrm{d} y \\
\leqslant & \frac{1}{\ln |x|}\left(\pi+\left\|f(\ln |f|)^{+}\right\|_{L^{1}\left(\mathbb{R}^{2}\right)}\right)+2\left(1+\frac{1}{\ln |x|}\right) \int_{\{|y|>|x|-1\}}|f(y)| \mathrm{d} y
\end{aligned}
$$

where we have used the well known inequality:

$$
a b \leqslant \mathrm{e}^{a}+b(\ln b-1) \leqslant \mathrm{e}^{a}+b(\ln b)^{+}, \quad \forall a \in \mathbb{R}, b>0
$$

with $a=\ln \frac{1}{|x-y|}$ and $b=|f(y)|$. Consequently, $\sigma_{1}(x) \rightarrow 0$ as $|x| \rightarrow+\infty$. Furthermore, for $x \in \mathbb{R}^{2}|x|>2 \rho$, we have

$$
\begin{aligned}
\left|\sigma_{2}(x)\right| & \leqslant \frac{1}{\ln |x|} \int_{\{|x-y|>1,|y|<\rho\}}\left(\left|\ln \left(\frac{|x-y|}{|x|}\right)\right|+\ln (\rho+1)\right)|f(y)| \mathrm{d} y \\
& \leqslant \frac{1}{\ln |x|} \ln (2(\rho+1))\|f\|_{L^{1}\left(\mathbb{R}^{2}\right)} \rightarrow 0, \quad \text { as }|x| \rightarrow+\infty .
\end{aligned}
$$

Finally,

$$
\begin{aligned}
\left|\sigma_{3}(x)\right| \leqslant & \frac{1}{\ln |x|} \int_{\{|x-y|>1, \rho<|y|<2|x|\}}(\ln |x-y|+\ln (1+|y|)+\ln |x|)|f(y)| \mathrm{d} y \\
& +\frac{1}{\ln |x|} \int_{\{|x-y|>1,|y|>2|x|\}}\left|\ln \left(\frac{|x-y|}{1+|y|}\right)\right||f(y)|+\int_{\{|y|>\rho\}}|f(y)| \mathrm{d} y \\
\leqslant & 4 \int_{\{|y|>\rho\}}|f(y)| \mathrm{d} y+\frac{3 \ln 3}{\ln |x|}\|f\|_{L^{1}\left(\mathbb{R}^{2}\right)} \rightarrow 4 \int_{\{|y|>\rho\}}|f(y)| \mathrm{d} y
\end{aligned}
$$

as $|x| \rightarrow+\infty$, for any fixed $\rho>1$. Thus, letting $\rho \rightarrow+\infty$, we obtain the desired conclusion. Now, suppose that (1.13) holds, then to establish (iii) it remains to show that

$$
\left|\int_{\mathbb{R}^{2}}(\ln |x-y|-\ln |x|) f(y) \mathrm{d} y\right|=\mathrm{O}(1)
$$


as $|x| \rightarrow+\infty$. As above, we are going to estimate the integral (1.19) over various regions. Firstly, note that for $|x|>1$ we have:

$$
\begin{aligned}
\left|\int_{\{|x-y|<1\}}(\ln |x-y|-\ln |x|) f(y) \mathrm{d} y\right| & \leqslant \int_{\{|x-y|<1\}} \ln \left(\frac{1}{|x-y|}\right)|f(y)| \mathrm{d} y+\int_{\{|x-y|<1\}} \ln |x||f(y)| \mathrm{d} y \\
& \leqslant \pi+\int_{\mathbb{R}^{2}}|f(y)|(\ln |f(y)|)^{+} \mathrm{d} y+\int_{\mathbb{R}^{2}} \ln (1+|y|)|f(y)| \mathrm{d} y:=C_{1} .
\end{aligned}
$$

On the other hand,

$$
\begin{aligned}
& \left|\int_{\{|x-y|>1\}}(\ln |x-y|-\ln |x|) f(y) \mathrm{d} y\right| \\
& \quad \leqslant \int_{\{|x-y|>1,|y|<|x| / 2\}}\left|\ln \left(\frac{|x-y|}{|x|}\right)\right||f(y)| \mathrm{d} y+\int_{\{|x-y|>1,|y|>|x| / 2\}}(\ln |x-y|+\ln |x|)|f(y)| \mathrm{d} y \\
& \quad \leqslant C_{2}\left(\|f\|_{L^{1}}+\int_{\mathbb{R}^{2}} \ln (1+|y|)|f(y)| \mathrm{d} y\right)
\end{aligned}
$$

for suitable $C_{2}>0$, and (1.19) follows.

Proof of Theorem 1.1. We start with the following:

\section{Claim 1.}

$$
\sup _{\mathbb{R}^{2}} u_{2} \leqslant \frac{c_{0}}{2}+\ln \left(\frac{1}{\pi}\left\|\mathrm{e}^{u_{2}}\right\|_{L^{1}}\right)
$$

Note that (1.20) immediately implies (1.5). To obtain (1.20), let us use the second equation in $(P)$ together with Green's representation formula, to derive that,

$$
\begin{aligned}
u_{2}(x) & \leqslant \frac{4 c_{0}}{2 \pi} \int_{\{|x-y|<r\}} \ln \left(\frac{r}{|x-y|}\right) \mathrm{d} y+\frac{1}{2 \pi r} \int_{\{|x-y|=r\}} u_{2}(y) \mathrm{d} \sigma \\
& =c_{0} r^{2}+\frac{1}{2 \pi r} \int_{\{|x-y|=r\}} u_{2}(y) \mathrm{d} \sigma,
\end{aligned}
$$

$\forall r>0$. Multiply both sides of (1.21) by $2 r$ and integrate over $[0,1]$ to obtain:

$$
u_{2}(x) \leqslant \frac{c_{0}}{2}+\frac{1}{\pi} \int_{B_{1}(x)} u_{2}(y) \mathrm{d} y .
$$

At this point, we can use Jensen's inequality to estimate,

$$
\frac{1}{\pi} \int_{B_{1}(x)} u_{2}(y) \mathrm{d} y \leqslant \ln \left(\frac{1}{\pi} \int_{B_{1}(x)} \mathrm{e}^{u_{2}(y)} \mathrm{d} y\right) \leqslant \ln \left(\frac{1}{\pi}\left\|\mathrm{e}^{u_{2}}\right\|_{L^{1}}\right),
$$

and conclude (1.20).

\section{Claim 2.}

$$
u_{1}^{+} \in L^{\infty}\left(\mathbb{R}^{2}\right) .
$$


To establish (1.22) note that $u_{1}(x) \rightarrow-\infty$ as $x \rightarrow z_{k}, k=1, \ldots, m$. So, we need to show that $u_{1}$ is bounded above outside a large ball which contains all points $z_{k}$ 's, $k=1, \ldots, m$. To this end note that, if $x:|x|>\max _{k=1, \ldots, m}\left|z_{k}\right|+2$, then

$$
\int_{B_{1}(x)} u_{1}^{+} \leqslant \int_{\mathbb{R}^{2}} \mathrm{e}^{u_{1}}
$$

and

$$
-\Delta u_{1}=4 g^{2} \mathrm{e}^{u_{1}}+g^{2} \mathrm{e}^{u_{2}} \quad \text { in } B_{1}(x)
$$

with $\mathrm{e}^{u_{2}} \in L^{1}\left(\mathbb{R}^{2}\right) \cap L^{\infty}\left(\mathbb{R}^{2}\right)$. So the argument given by Brezis and Merle [6] in the proof of Theorem 2 applies word by word and yields to the estimate:

$$
\max _{B_{1 / 4}(x)} u_{1} \leqslant C
$$

with a suitable constant $C>0$ independent of $x$.

To proceed further, let

$$
w_{2}(x)=\frac{g^{2}}{2 \pi} \int_{\mathbb{R}^{2}}(\ln |x-y|-\ln (1+|y|)) \mathrm{e}^{u_{2}(y)} \mathrm{d} y .
$$

Hence, by Lemma 1.1, we have

$$
w_{2}(x) \leqslant \sigma_{2} \ln (|x|+1), \quad \forall x \in \mathbb{R}^{2},
$$

and

$$
\frac{w_{2}(x)}{\ln |x|} \rightarrow \sigma_{2}, \quad \text { as }|x| \rightarrow+\infty .
$$

Define,

$$
u_{0}(x)=\sum_{k=1}^{m} \ln \left|x-z_{k}\right|^{2 n_{k}}
$$

and note that

$$
u_{1}-u_{0} \in L_{\mathrm{loc}}^{\infty}\left(\mathbb{R}^{2}\right) \text {. }
$$

Decompose:

$$
u_{1}(x)=u_{0}(x)-w_{2}(x)+v_{1}(x)
$$

so that $v_{1}(x)$ satisfies:

$$
v_{1} \in L_{\mathrm{loc}}^{\infty}\left(\mathbb{R}^{2}\right) ; \quad-\Delta v_{1}(x)=4 g^{2} \mathrm{e}^{u_{1}(x)}=4 g^{2} \prod_{k=1}^{m}\left|x-z_{k}\right|^{2 n_{k}} \mathrm{e}^{-w_{2}(x)} \mathrm{e}^{v_{1}},
$$

and

$$
\frac{v_{1}^{+}(x)}{\ln (|x|+1)} \in L^{\infty}\left(\mathbb{R}^{2}\right)
$$

Therefore, we may use Corollary 1.1 to derive that

$$
-v_{1}(x) \leqslant \sigma_{1}(\ln (|x|+1))+C
$$


for suitable $C>0$, and

$$
\frac{v_{1}(x)}{\ln |x|} \rightarrow-\sigma_{1}, \quad \text { as }|x| \rightarrow+\infty .
$$

In virtue of (1.25), (1.26) and (1.27), we conclude:

$$
u_{1}(x) \geqslant\left(2 N-\left(\sigma_{1}+\sigma_{2}\right)\right) \ln |x|-C, \quad \text { as }|x| \rightarrow+\infty
$$

and

$$
\frac{u_{1}(x)}{\ln |x|} \rightarrow 2 N-\left(\sigma_{1}+\sigma_{2}\right) \quad \text { as }|x| \rightarrow+\infty
$$

So, (1.2) and (1.3) are established. Consequently, we must have that $\sigma_{1}+\sigma_{2}>2(N+1)$ and

$$
\int_{\mathbb{R}^{2}} \ln (1+|y|) \mathrm{e}^{u_{1}(y)} \mathrm{d} y<+\infty .
$$

Thus, we can use part (iii) of Lemma 1.1 together with Corollary 1.1 to conclude that,

$$
v_{1}(x)=\sigma_{1} \ln \frac{1}{|x|}+\mathrm{O}(1), \quad \text { as }|x| \rightarrow+\infty .
$$

Finally, suppose that (1.6) holds, then we are in position to apply Corollary 1.1 to the function $v_{2}(x)=u_{2}(x)+$ $c_{0}\left|x-x_{0}\right|^{2}$ with $f(x)=\frac{g^{2}}{2 \cos ^{2} \theta} \mathrm{e}^{u_{2}}+2 g^{2} \mathrm{e}^{u_{1}}$, and conclude that

$$
\frac{u_{2}(x)+c_{0}\left|x-x_{0}\right|^{2}}{\ln |x|} \rightarrow \frac{1}{2}\left(\sigma_{1}+\frac{\sigma_{2}}{\cos ^{2} \theta}\right) \quad \text { as }|x| \rightarrow+\infty .
$$

In particular, this implies that $u_{2}$ admits exponential decay at infinity. Thus, $\int_{\mathbb{R}^{2}} \ln (1+|y|) \mathrm{e}^{u_{2}(y)} \mathrm{d} y<+\infty$, and taking into account (1.33), we derive:

$$
w_{2}(x)=\sigma_{2} \ln |x|+\mathrm{O}(1), \quad \text { as }|x| \rightarrow+\infty,
$$

and

$$
u_{2}(x)=-c_{0}\left|x-x_{0}\right|^{2}+\frac{1}{2}\left(\sigma_{1}+\frac{\sigma_{2}}{\cos ^{2} \theta}\right) \ln |x|+\mathrm{O}(1)
$$

as $|x| \rightarrow+\infty$. Moreover, from (1.29), (1.34) and (1.35) we conclude

$$
u_{1}(x)=\sum_{k=1}^{m} \ln \left|z-z_{k}\right|^{2 n_{k}}-\left(\sigma_{1}+\sigma_{2}\right) \ln |x|+\mathrm{O}(1) \quad \text { as }|x| \rightarrow \infty,
$$

and Theorem 1.1 is established.

Remark 1.1. We point out that, as in Lemma 1.3 of [8], it is possible to sharpen the asymptotic behavior (1.7) and (1.8) and prove that

$$
\begin{aligned}
& \frac{\partial u_{1}}{\partial r} \rightarrow 2 N-\left(\sigma_{1}+\sigma_{2}\right), \quad \frac{\partial u_{1}}{\partial \theta} \rightarrow 0, \\
& r \frac{\partial}{\partial r}\left(u_{2}+c_{0}\left|x-x_{0}\right|^{2}\right) \rightarrow \frac{1}{2}\left(\sigma_{1}+\frac{\sigma_{2}}{\cos ^{2} \theta}\right), \quad \frac{\partial}{\partial \theta}\left(u_{2}+c_{0}\left|x-x_{0}\right|^{2}\right) \rightarrow 0
\end{aligned}
$$

uniformly, as $r=|x| \rightarrow+\infty$, with $(r, \theta)$ polar coordinates in $\mathbb{R}^{2}$. 
In the framework of Theorem 1.1, Spruck and Yang in [14] have constructed two families of solutions satisfying the asymptotic behavior (1.7), (1.8) (with $x_{0}=0$ ) and corresponding values $\sigma_{1}, \sigma_{2}$ free to vary as follows:

1. for the first family: $\sigma_{1} \in(0,4)$ and $\sigma_{1}+\sigma_{2} \in(2(N+2),+\infty)$;

2. for the second family: $\sigma_{1} \in(0,2)$ and $2 \sigma_{1}+\sigma_{2} \in(2(N+1),+\infty)$.

In particular, Spruck-Yang's construction allows one to obtain a solution pair for $(P)$ with $\sigma_{1}+\sigma_{2}$ equals to any prescribed value in the interval $(2(N+1),+\infty)$. So, $(1.4)$ is sharp. On the other hand, their construction always gives, $\sigma_{2}>2 N$, while $\sigma_{1}$ can take values as small as wanted. For instance, according to Spruck-Yang's result, it is possible to exhibit a family of solutions for $(P)$ such that $\sigma_{1}=\mathrm{o}(1)$, while $\sigma_{2}$ can take any fixed value in $(2(N+1),+\infty)$. Clearly, those solutions deny any sort of "quantization" property to the corresponding flux $(0.5)$.

The aim of this paper is to show that the situation is quite different in case we consider solutions of $(P)$ that admit the asymptotic behavior (1.7), (1.8) with $\sigma_{2}$ small. We see that in this case $\sigma_{1}$ may not be free to take any value in the interval $(2(N+1),+\infty)$, as $(1.4)$ would imply. In fact, in case all vortex points coincide, we see that $\sigma_{1}$ must remain close to the value $4(N+1)$ and no other value is allowed. Thus solution of $(P)$ with $\sigma_{2}$ small are very interesting, as they seem to restore a sort of "quantization" property for the flux in (0.5). We are going to construct such class of solutions. To this purpose, we consider the following function spaces introduced by Chae and Imanuvilov in [7].

For given $\alpha>0$, let

$$
X_{\alpha}=\left\{u \in L_{\text {loc }}^{2}\left(\mathbb{R}^{2}\right): \int_{\mathbb{R}^{2}}\left(1+|x|^{2+\alpha}\right)|u(x)|^{2} \mathrm{~d} x<\infty\right\},
$$

which defines a Hilbert space equipped with the scalar product

$$
(u, v)=\int_{\mathbb{R}^{2}}\left(1+|x|^{2+\alpha}\right) u v .
$$

Denote with $\|\cdot\|_{X_{\alpha}}$ the corresponding norm on $X_{\alpha}$. Also let

$$
Y_{\alpha}=\left\{u \in W_{\mathrm{loc}}^{2,2}\left(\mathbb{R}^{2}\right): \Delta u \in X_{\alpha}, \frac{u}{(1+|x|)^{1+\alpha / 2}} \in L^{2}\left(\mathbb{R}^{2}\right)\right\}
$$

It defines a Hilbert space with corresponding natural scalar product and norm:

$$
\|u\|_{Y_{\alpha}}^{2}=\|\Delta u\|_{X_{\alpha}}^{2}+\left\|\frac{u(x)}{1+|x|^{1+\alpha / 2}}\right\|_{L^{2}\left(\mathbb{R}^{2}\right)}^{2} .
$$

We refer to [7] for the relevant properties of those spaces. In particular, from [7] we recall,

Proposition 1.1. (a) $X_{\alpha} \hookrightarrow L^{1}\left(\mathbb{R}^{2}\right)$ continuously, $Y_{\alpha} \hookrightarrow C_{\text {loc }}^{0}\left(\mathbb{R}^{2}\right)$.

(b) If $\alpha \in(0,1)$ and $u \in Y_{\alpha}$ is harmonic, then $u$ is constant.

(c) There exists a constant $C_{1}>0$ such that for all $u \in Y_{\alpha}$

$$
|u(x)| \leqslant C_{1}\|u\|_{Y_{\alpha}}\left((\ln |x|)^{+}+1\right), \quad \forall x \in \mathbb{R}^{2} .
$$


Notice that assumption (1.6) is motivated by considering solutions of $(P)$ in the space $Y_{\alpha} \times Y_{\alpha}$. In fact, as an easy consequence of Theorem 1.1, it follows:

Corollary 1.2. Under the assumptions of Theorem 1.1 we have

(i) $U_{1}:=u_{1}-u_{0} \in Y_{\alpha}, U_{2}:=u_{2}+c_{0}\left|x-x_{0}\right|^{2} \in Y_{\alpha}$ and

$$
\mathrm{e}^{u_{1}} \in X_{\alpha} \quad \text { for every } \alpha \in\left(0,2\left(\sigma_{1}+\sigma_{2}-2(N+1)\right)\right)
$$

(ii) $\frac{1}{2} U_{1}+U_{2} \in Y_{\alpha}$ and $\mathrm{e}^{u_{2}} \in X_{\alpha}, \forall \alpha>0$.

To complement the situation analyzed by Spruck and Yang in [14], we consider solutions of ( $P$ ) with $\sigma_{2} \leqslant 2 N$. In this case, the following interesting estimate holds, when all vortex points coincide.

Lemma 1.2. Let $\left(u_{1}, u_{2}\right)$ be solutions of $(P)$ with $z_{1}=z_{2}=\cdots=z_{m}$ and such that $e^{u_{2}} \in X_{\alpha}$ with $\alpha>2$ and $\sigma_{2} \leqslant 2 N$. For any $q \in\left(2, \frac{\alpha+4}{3}\right)$ there exists a constant $C_{q}=C\left(q, c_{0}, \alpha\right)>0$ such that

$$
\left|\sigma_{1}+2 \sigma_{2}-4(N+1)\right| \leqslant C_{q} \sigma_{2}^{(q-2) / q}\left\|\mathrm{e}^{u_{2}}\right\|_{X_{\alpha}}^{2 / q} .
$$

Remark 1.2. Clearly, estimate (1.37) bears interesting consequences in case $\left\|\mathrm{e}^{u_{2}}\right\|_{X_{\alpha}}$ (and hence $\sigma_{2}$ ) is small, as it forces $\sigma_{1}$ to remain close to the value $4(N+1)$. Such a situation can actually occur, as we are going to construct family of solutions $\left(u_{1, \varepsilon}, u_{2, \varepsilon}\right)$ for $(P)$ (when all vortex points coincide) such that, as $\varepsilon \rightarrow 0,\left\|\mathrm{e}^{u_{2, \varepsilon}}\right\|_{X_{\alpha}}=\mathrm{o}(1)$, so

$$
\begin{aligned}
\sigma_{2, \varepsilon} & =\frac{g^{2}}{2 \pi} \int_{\mathbb{R}^{2}} \mathrm{e}^{u_{2, \varepsilon}}=\mathrm{o}(1), \\
\sigma_{1, \varepsilon} & =\frac{2 g^{2}}{\pi} \int_{\mathbb{R}^{2}} \mathrm{e}^{u_{1, \varepsilon}}=4(N+1)+\mathrm{o}(1) .
\end{aligned}
$$

Proof. Without loss of generality suppose that $z_{1}=\cdots=z_{m}=0$. Thus, by (1.30) we have:

$$
v_{1} \in L_{\mathrm{loc}}^{\infty}\left(\mathbb{R}^{2}\right), \quad-\Delta v_{1}=4 g^{2}|x|^{2 N} \mathrm{e}^{-w_{2}(x)} \mathrm{e}^{v_{1}}
$$

with $w_{2}$ as defined in (1.24). Since $\mathrm{e}^{u_{2}} \in X_{\alpha}$, then we also have $\int_{\mathbb{R}^{2}} \ln (1+|x|) \mathrm{e}^{u_{2}(x)} \mathrm{d} x<+\infty$, and in view of Lemma 1.2, we derive

$$
w_{2}(x)=\sigma_{2} \ln (|x|+1)+\mathrm{O}(1) .
$$

Consequently, setting $R(x)=4 g^{2}|x|^{2 N} \mathrm{e}^{-w_{2}(x)}$, we see that

$$
R(x)=\mathrm{O}\left(|x|^{2 N-\sigma_{2}}\right),
$$

and, by assumption, $2 N-\sigma_{2} \geqslant 0$. Furthermore,

$$
\int_{\mathbb{R}^{2}} R(x) \mathrm{e}^{v_{1}(x)} \mathrm{d} x=4 g^{2} \int_{\mathbb{R}^{2}} \mathrm{e}^{u_{1}}=2 \pi \sigma_{1}
$$

and, from (1.40), we get $\int_{\mathbb{R}^{2}}\left(1+|x|^{2 N-\sigma_{2}}\right) \mathrm{e}^{v_{1}}<+\infty$. Thus, we are in position to apply Theorem 1 (when $2 N=\sigma_{2}$ ), or Theorem 2 (when $2 N>\sigma_{2}$ ) of Chen and Li [8], to conclude: 


$$
\begin{aligned}
\pi \sigma_{1}\left(\sigma_{1}-4\right) & =\int_{\mathbb{R}^{2}} x \cdot \nabla R(x) \mathrm{e}^{v_{1}(x)} \mathrm{d} x \\
& =4 g^{2}\left(2 N \int_{\mathbb{R}^{2}} R(x) \mathrm{e}^{v_{1}}-\int_{\mathbb{R}^{2}} x \cdot \nabla w_{2}(x) R(x) \mathrm{e}^{v_{1}}\right) \\
& =4 \pi N \sigma_{1}-2 \pi \sigma_{2} \sigma_{1}-4 g^{2} \int_{\mathbb{R}^{2}}\left(x \cdot \nabla w_{2}(x)-\sigma_{2}\right) R(x) \mathrm{e}^{v_{1}} .
\end{aligned}
$$

That is,

$$
\left|\sigma_{1}+2 \sigma_{2}-4(N+1)\right| \leqslant \frac{4 g^{2}}{\pi \sigma_{1}} \int_{\mathbb{R}^{2}}\left|x \cdot \nabla w_{2}(x)-\sigma_{2}\right| R(x) \mathrm{e}^{v_{1}(x)} \mathrm{d} x .
$$

On the other hand, by (1.24) we have:

$$
\begin{aligned}
\left|x \cdot \nabla w_{2}(x)-\sigma_{2}\right| & \leqslant \frac{g^{2}}{2 \pi} \int_{\mathbb{R}^{2}} \frac{|y|}{|x-y|} \mathrm{e}^{u_{2}(y)} \mathrm{d} y \leqslant \frac{g^{2}}{2 \pi} \int_{\mathbb{R}^{2}} \frac{(1+|y|)^{\frac{2+\alpha}{q}} \mathrm{e}^{u_{2}(y)}}{|x-y|(1+|y|)^{\frac{2+\alpha}{q}}-1} \mathrm{~d} y \\
& \leqslant \frac{g^{2}}{2 \pi}\left(\int_{\mathbb{R}^{2}}(1+|y|)^{2+\alpha} \mathrm{e}^{q u_{2}} \mathrm{~d} y\right)^{\frac{1}{q}}\left(\int_{\mathbb{R}^{2}} \frac{\mathrm{d} y}{|x-y|^{\frac{q}{q-1}}(1+|y|)^{\frac{2+\alpha-q}{q-1}}}\right)^{\frac{q-1}{q}} \\
& \leqslant \frac{g^{2}}{2 \pi}\left\|\mathrm{e}^{u_{2}}\right\|_{L^{\infty}\left(\mathbb{R}^{2}\right)}^{\frac{q-2}{q}}\left\|\mathrm{e}^{u_{2}}\right\|_{X_{\alpha}}^{2 / q}\left(\int_{\{|x-y|<1\}} \frac{\mathrm{d} y}{|x-y|^{\frac{q}{q-1}}}+\int_{\{|x-y| \geqslant 1\}} \frac{\mathrm{d} y}{(1+|y|)^{\frac{2+\alpha-1}{q-1}}}\right)^{\frac{q-1}{q}} .
\end{aligned}
$$

At this point, the desired estimate (1.37) follows, by means of (1.5), and the observation that, by the choice of $q \in\left(2, \frac{\alpha+4}{3}\right)$ we have $1<\frac{q}{q-1}<2<\frac{2+\alpha-q}{q-1}$. Thus,

$$
\left|x \cdot \nabla w_{2}(x)-\sigma_{2}\right| \leqslant C_{1, q}\left\|\mathrm{e}^{u_{1}}\right\|_{L^{1}\left(\mathbb{R}^{2}\right)}^{\frac{q-2}{q}}\left\|\mathrm{e}^{u_{2}}\right\|_{X_{\alpha}}^{\frac{2}{q}}
$$

with a suitable constant $C_{1, q}>0$ independent of $x \in \mathbb{R}^{2}$. Hence, we can use (1.43) in (1.42), and by (1.41), obtain (1.37).

\section{Existence result}

We devote this section to the construction of a four-parameter family of solutions for $(P)$ in case all vortex points coincide, such that (1.38) and (1.39) hold. Without loss of generality we take all vortex points to coincide with the origin and obtain,

Theorem 2.1. Let $z_{1}=z_{2}=\cdots=z_{m}=0$, there exists $\varepsilon_{0}>0$ sufficiently small such that problem $(\mathcal{P})$ admits a five-parameters family of solutions $\left(u_{1, \boldsymbol{\alpha}}^{\varepsilon}, u_{2, \boldsymbol{\alpha}}^{\varepsilon}\right)$ with $\varepsilon \in\left(-\varepsilon_{0}, \varepsilon_{0}\right), \boldsymbol{\alpha} \in \mathbb{R}^{4}$ and $|\boldsymbol{\alpha}|<\varepsilon_{0}$ satisfying:

$$
\frac{1}{\varepsilon}\left\|\mathrm{e}^{u_{2, \alpha}^{\varepsilon}}\right\|_{X_{\alpha}}=\mathrm{O}(1) \quad \forall \alpha>0, \quad \frac{2 g^{2}}{\pi} \int_{\mathbb{R}^{2}} \mathrm{e}^{u_{1, \alpha}^{\varepsilon}}=4(N+1)+\varepsilon \mathrm{O}(1) \quad \text { as } \varepsilon \rightarrow 0 \text {, }
$$


(ii)

$$
\begin{aligned}
& u_{1, \boldsymbol{\alpha}}^{\varepsilon}(x)=-\left[2(N+2)+\varepsilon\left(\frac{1}{2 c_{0}}\left(\frac{(N+1) !}{c_{0}^{N+1}}-1\right)+\beta_{1, \boldsymbol{\alpha}}^{\varepsilon}\right)\right] \ln |x|+\mathrm{O}(1), \\
& u_{2, \boldsymbol{\alpha}}^{\varepsilon}(x)=-c_{0}|x|^{2}+\left(2(N+1)+\beta_{2, \boldsymbol{\alpha}}^{\varepsilon}\right) \ln |x|+\ln \varepsilon+\mathrm{O}(1) \quad \text { as }|x| \rightarrow \infty
\end{aligned}
$$

where $\beta_{1, \alpha}^{\varepsilon} \rightarrow 0, \beta_{2, \alpha}^{\varepsilon} \rightarrow 0$ as $\varepsilon \rightarrow 0,|\boldsymbol{\alpha}| \rightarrow 0$, and $\mathrm{O}(1)$ denotes a quantity bounded uniformly in $(\varepsilon, \boldsymbol{\alpha})$.

In order to obtain Theorem 2.1, we shall establish a more general existence result concerning the problem:

$$
\left(\mathrm{P}_{\varepsilon}\right)\left\{\begin{array}{l}
-\Delta u_{1}=4 g^{2} \mathrm{e}^{u_{1}}+g^{2} \mathrm{e}^{u_{2}}-4 \pi \sum_{k=1}^{m} n_{k} \delta\left(z-\varepsilon z_{k}\right), \\
\Delta u_{2}=\frac{g^{2}}{2 \cos ^{2} \theta}\left(\mathrm{e}^{u_{2}}-\phi_{0}^{2}\right)+2 g^{2} \mathrm{e}^{u_{1}}, \\
\int_{\mathbb{R}^{2}} \mathrm{e}^{u_{1}}<\infty, \quad \int_{\mathbb{R}^{2}} \mathrm{e}^{u_{2}}<\infty
\end{array}\right.
$$

with $z_{1}, \ldots, z_{m}$ arbitrarily given points in $\mathbb{R}^{2}$ and small $\varepsilon \in \mathbb{R}$. For this purpose, it is convenient to introduce complex notation and identify the pair $x=\left(x_{1}, x_{2}\right) \in \mathbb{R}^{2}$ with the complex number $z=x+i y \in \mathbb{C}$. Let

$$
f_{\varepsilon}(z)=(N+1) \prod_{k=1}^{m}\left(z-\varepsilon z_{k}\right)^{n_{k}}, \quad \text { and } \quad F_{\varepsilon}(z)=\int_{0}^{z} f_{\varepsilon}(\xi) \mathrm{d} \xi .
$$

For any $a \in \mathbb{C}$, define,

$$
\eta_{\varepsilon, a}(z)=\frac{8\left|f_{\varepsilon}(z)\right|^{2}}{\left(1+\left|F_{\varepsilon}(z)+a\right|^{2}\right)^{2}},
$$

and consider the radial function $\rho=\rho(r)$ so that,

$$
\rho(|z|):=\eta_{\varepsilon=0, a=0}(z)=\frac{8(N+1)^{2}|z|^{2 N}}{\left(1+|z|^{2 N+2}\right)^{2}} .
$$

As well known (e.g., [12]), $\forall \varepsilon \geqslant 0$ and $\forall a \in \mathbb{C}$ we have

$$
\begin{aligned}
& -\Delta \ln \eta_{\varepsilon, a}=\eta_{\varepsilon, a}-4 \pi \sum_{k=1}^{m} n_{k} \delta\left(z-\varepsilon z_{k}\right), \quad \text { in } \mathbb{R}^{2}, \\
& \int_{\mathbb{R}^{2}} \eta_{\varepsilon, a}=8 \pi(N+1) .
\end{aligned}
$$

We shall look for solutions of $\left(\mathrm{P}_{\varepsilon}\right)$ with a specific structure. Namely, we set

$$
\begin{aligned}
& u_{1}(z)=\ln \eta_{\varepsilon, a}(z)+\varepsilon\left(w_{1}(z)+v_{1}(z)\right)-2 \ln 2 g, \\
& u_{2}(z)=-c_{0}|z|^{2}+w_{2}(z)+\ln \varepsilon-2 \ln g+v_{2}(z),
\end{aligned}
$$

where $w_{2}$ is the radial function given by

$$
w_{2}(r)=\ln \left(1+r^{2 N+2}\right),
$$

and so, it satisfies

$$
\Delta w_{2}=\frac{\rho}{2},
$$


while $w_{1}$ is the radial solution in $Y_{\alpha}, \alpha \in\left(0, \frac{1}{2}\right)$ of the equation:

$$
\Delta w+\rho w+\mathrm{e}^{-c_{0}|z|^{2}}\left(1+|z|^{2 N+2}\right)=0 \quad \text { in } \mathbb{R}^{2},
$$

as constructed in Lemma 2.1 of [7]. Consequently, relative to the unknowns $\left(v_{1}, v_{2}\right)$, problem $\left(\mathrm{P}_{\varepsilon}\right)$ becomes:

$$
\left(\mathrm{P}_{\varepsilon}^{\prime}\right) \quad\left\{\begin{array}{l}
-\Delta v_{1}=\mathrm{e}^{-c_{0}|z|^{2}+w_{2}+v_{2}}+\eta_{\varepsilon, a} \frac{\left(\mathrm{e}^{\varepsilon\left(v_{1}+w_{1}\right)}-1\right)}{\varepsilon}-\rho w_{1}-\mathrm{e}^{-c_{0}|z|^{2}+w_{2}}, \\
\Delta v_{2}=\frac{\varepsilon}{2 \cos ^{2} \theta} \mathrm{e}^{-c_{0}|z|^{2}+w_{2}+v_{2}}+\frac{1}{2} \eta_{\varepsilon, a} \mathrm{e}^{\varepsilon\left(v_{1}+w_{1}\right)}-\frac{\rho}{2},
\end{array}\right.
$$

as it follows by direct computations. Notice that, by continuity problem $\left(\mathrm{P}_{\varepsilon}^{\prime}\right)$ may be considered also at $\varepsilon=0$, and for $a=0,\left(\mathrm{P}_{\varepsilon=0}^{\prime}\right)$ admits the (trivial) solution $v_{1}=v_{2}=0$. For $\varepsilon$ small we aim to construct solutions for $\left(\mathrm{P}_{\varepsilon}^{\prime}\right)$ which "bifurcate" from such trivial solution. To this purpose, we start by collecting some useful properties of the function $w_{1}$, which will be established in Appendix A.

Lemma 2.1. Problem (2.7) admits a radial solution $w_{1}$ such that

$$
w_{1} \in Y_{\alpha}, \quad \forall \alpha \in\left(0, \frac{1}{2}\right)
$$

$$
\begin{array}{ll} 
& w_{1}(r)=\frac{1}{2 c_{0}}\left(1-\frac{(N+1) !}{c_{0}^{N+1}}\right) \ln r+\mathrm{O}(1), \quad \text { as } r \rightarrow+\infty ; \\
& \frac{r \mathrm{~d} w_{1}}{\mathrm{~d} r} \rightarrow \frac{1}{2 c_{0}}\left(1-\frac{(N+1) !}{c_{0}^{N+1}}\right), \quad \text { as } r \rightarrow+\infty ; \\
& \int_{0}^{+\infty}\left(-2 \rho(r) w_{1}(r) \frac{r^{2(N+1)}}{\left(1+r^{2(N+1)}\right)^{2}}+\frac{\mathrm{e}^{-c_{0} r^{2} r^{2(N+1)}}}{1+r^{2(N+1)}}\right) r \mathrm{~d} r=\frac{1}{2 c_{0}} .
\end{array}
$$

From now on, the function $w_{1}$ in (2.5) is chosen according to Lemma 2.1.

For fixed $\alpha>0$ define the operator

$$
P: Y_{\alpha}^{2} \times \mathbb{C} \times \mathbb{R} \rightarrow X_{\alpha}^{2}
$$

by setting $P=\left(P_{1}, P_{2}\right)$ with

$$
\begin{aligned}
& P_{1}\left(v_{1}, v_{2}, a, \varepsilon\right)=\Delta v_{1}+\mathrm{e}^{-c_{0}|z|^{2}+w_{2}+v_{2}}+\eta_{\varepsilon, a} \frac{\left(\mathrm{e}^{\varepsilon\left(v_{1}+w_{1}\right)}-1\right)}{\varepsilon}-\rho w_{1}-\mathrm{e}^{-c_{0}|z|^{2}+w_{2}}, \\
& P_{2}\left(v_{1}, v_{2}, a, \varepsilon\right)=\Delta v_{2}-\frac{\varepsilon}{2 \cos ^{2} \theta} \mathrm{e}^{-c_{0}|z|^{2}+v_{2}+w_{2}}-\frac{1}{2} \eta_{\varepsilon, a} \mathrm{e}^{\varepsilon\left(v_{1}+w_{1}\right)}-\frac{\rho}{2}
\end{aligned}
$$

and extended by continuity at $\varepsilon=0$. Thus, $P(0,0,0,0)=(0,0)$, and finding a solution for problem $\left(\mathrm{P}_{\varepsilon}^{\prime}\right)$ is now reduced to finding a small $\varepsilon_{0}>0$ and an implicit function

$$
\varepsilon \mapsto\left(v_{1, \varepsilon}, v_{2, \varepsilon}, a_{\varepsilon}\right):\left(-\varepsilon_{0}, \varepsilon_{0}\right) \rightarrow Y_{\alpha}^{2} \times \mathbb{C}
$$

satisfying $P\left(v_{1, \varepsilon}, v_{2, \varepsilon}, a_{\varepsilon}, \varepsilon\right)=0, \forall \varepsilon \in\left(-\varepsilon_{0}, \varepsilon_{0}\right)$. To this end, let $a=a_{1}+i a_{2}$ and observe that,

$$
\left.\frac{\partial \eta_{\varepsilon, a}(z)}{\partial a_{1}}\right|_{a=0, \varepsilon=0}=-4 \rho \varphi_{+},\left.\quad \frac{\partial \eta_{\varepsilon, a}(z)}{\partial a_{2}}\right|_{a=0, \varepsilon=0}=-4 \rho \varphi_{-},
$$


where, in polar coordinates,

$$
\varphi_{+}(r, \theta)=\frac{r^{N+1} \cos (N+1) \theta}{1+r^{2 N+2}}, \quad \varphi_{-}(r, \theta)=\frac{r^{N+1} \sin (N+1) \theta}{1+r^{2 N+2}} .
$$

Therefore, the linearized operator $P_{\left(v_{1}, v_{2}, a\right)}^{\prime}=\left(P_{1,\left(v_{1}, v_{2}, a\right)}^{\prime}, P_{2,\left(v_{1}, v_{2}, a\right)}^{\prime}\right)$ of $P$ at $(0,0,0,0)$ may be easily computed as given by,

$$
\begin{aligned}
& P_{1,\left(v_{1}, v_{2}, a\right)}^{\prime}(0,0,0,0)\left[\psi_{1}, \psi_{2}, b\right]=\Delta \psi_{1}+\rho \psi_{1}+\mathrm{e}^{-c_{0}|z|^{2}+w_{2}} \psi_{2}-4 \rho w_{1} \varphi_{+} b_{1}-4 \rho w_{1} \varphi_{-} b_{2}, \\
& P_{2,\left(v_{1}, v_{2}, a\right)}^{\prime}(0,0,0,0)\left[\psi_{1}, \psi_{2}, b\right]=\Delta \psi_{2}+2 \rho \varphi_{+} b_{1}+2 \rho \varphi_{-} b_{2} .
\end{aligned}
$$

Set $P_{\left(v_{1}, v_{2}, a\right)}^{\prime}(0,0,0,0)=A$, we have:

Proposition 2.1. Let $\alpha \in\left(0, \frac{1}{2}\right)$, then the operator $A: Y_{\alpha}^{2} \times \mathbb{C} \rightarrow X_{\alpha}^{2}$ is onto, and

$$
\operatorname{Ker} A=\operatorname{Span}\left\{\left(\varphi_{+}, 0\right),\left(\varphi_{-}, 0\right),\left(\varphi_{0}, 0\right),\left(\omega_{1}, 1\right)\right\} \times\{\mathbf{0}\}
$$

where $\varphi_{0}$ is the radial function:

$$
\varphi_{0}(r)=\frac{1-r^{2(N+1)}}{1+r^{2(N+1)}}, \quad r=|z| .
$$

Proof. In order to prove Proposition 2.1, we recall the following result established in [7] for the operator

$$
L=\Delta+\rho: Y_{\alpha} \rightarrow X_{\alpha} .
$$

Namely, if $\alpha \in\left(0, \frac{1}{2}\right)$, then

$$
\operatorname{Ker} L=\operatorname{Span}\left\{\varphi_{+}, \varphi_{-}, \varphi_{0}\right\}
$$

(see Lemma 2.4 in [7]) and

$$
\operatorname{Im} L=\left\{f \in X_{\alpha}: \int_{\mathbb{R}^{2}} f \varphi_{ \pm}=0\right\}
$$

(see Proposition 2.2 in [7]). Now, let $\left(f_{1}, f_{2}\right) \in X_{\alpha}^{2}$, we seek $\left(\psi_{1}, \psi_{2}, b\right) \in Y_{\alpha}^{2} \times \mathbb{C}, b=b_{1}+\mathrm{i} b_{2}$ such that

$$
\Delta \psi_{1}+\rho \psi_{1}+\mathrm{e}^{-c_{0}|z|^{2}+w_{2}} \psi_{2}-4 \rho w_{1} \varphi_{+} b_{1}-4 \rho w_{1} \varphi_{-} b_{2}=f_{1}
$$

and

$$
\Delta \psi_{2}+2 \rho \varphi_{+} b_{1}+2 \rho \varphi_{-} b_{2}=f_{2} .
$$

We start by considering (2.12). Decompose

$$
\psi_{2}=\phi_{2}+2 d_{1} \varphi_{+}+2 d_{2} \varphi_{-}
$$

where the constants $d_{1}, d_{2}$ will be specified later, and $\phi_{2} \in Y_{\alpha}$ :

$$
\int_{\mathbb{R}^{2}} \Delta \phi_{2} \varphi_{ \pm}=0
$$

Therefore, recalling that $\Delta \varphi_{ \pm}+\rho \varphi_{ \pm}=0,(2.12)$ holds if and only if

$$
\Delta \phi_{2}=2\left(d_{1}-b_{1}\right) \rho \varphi_{+}+2\left(d_{2}-b_{2}\right) \rho \varphi_{-}+f_{2} .
$$


In order to meet the orthogonality condition (2.14), necessarily:

$$
\int_{\mathbb{R}^{2}} f_{2} \varphi_{+}+2\left(d_{1}-b_{1}\right) \int_{\mathbb{R}^{2}} \rho \varphi_{+}^{2}=0 ; \quad \int_{\mathbb{R}^{2}} f_{2} \varphi_{-}+2\left(d_{2}-b_{2}\right) \int_{\mathbb{R}^{2}} \rho \varphi_{-}^{2}=0 .
$$

By elementary calculations, we see that

$$
\int_{\mathbb{R}^{2}} \rho \varphi_{ \pm}^{2}=\frac{2}{3} \pi(N+1) .
$$

So (2.16) requires the choise:

$$
d_{1}=b_{1}-\frac{3}{4 \pi(N+1)} \int_{\mathbb{R}^{2}} f_{1} \varphi_{+}, \quad d_{2}=b_{2}-\frac{3}{4 \pi(N+1)} \int_{\mathbb{R}^{2}} f_{2} \varphi_{-} .
$$

Hence, inserting (2.17) into (2.15), and letting

$$
f=f_{2}-\left(\frac{3}{2 \pi(N+1)} \int_{\mathbb{R}^{2}} f_{2} \varphi_{+}\right) \rho \varphi_{+}-\left(\frac{3}{2 \pi(N+1)} \int_{\mathbb{R}^{2}} f_{2} \varphi_{-}\right) \rho \varphi_{-} \in X_{\alpha},
$$

it suffices to take

$$
\phi_{2}(x)=\frac{1}{2 \pi} \int_{\mathbb{R}^{2}} \ln |x-y| f(y) \mathrm{d} y,
$$

in order to obtain a solution for (2.14)-(2.15) in $Y_{\alpha}$.

Thus $\psi_{2}$ in (2.13) is determined by (2.17) and (2.18). Now, insert such $\psi_{2}$ into (2.11) to obtain:

$$
\Delta \psi_{1}+\rho \psi_{1}=g_{1}-2\left(\mathrm{e}^{-c_{0}|z|^{2}+w_{2}}-2 \rho w_{1}\right)\left(b_{1} \varphi_{+}+b_{2} \varphi_{-}\right)
$$

with

$$
g_{1}=f_{1}-\mathrm{e}^{-c_{0}|z|^{2}+w_{2}}\left[\phi_{2}-\frac{3}{2 \pi(N+1)}\left(\varphi_{+} \int_{\mathbb{R}^{2}} f_{2} \varphi_{+}+\varphi_{-} \int_{\mathbb{R}^{2}} f_{2} \varphi_{-}\right)\right],
$$

and $\phi_{2}$ given in (2.18). Next, we are going to choose $b_{1}$ and $b_{2}$ in order to insure that the right hand side of (2.19) satisfies to the orthogonality conditions required by (2.10). Namely, we impose:

$$
\begin{aligned}
& \int_{\mathbb{R}^{2}} g_{1} \varphi_{+}-2 b_{1} \int_{\mathbb{R}^{2}}\left(\mathrm{e}^{-c_{0}|z|^{2}+w_{2}}-2 \rho w_{1}\right) \varphi_{+}^{2}=0, \\
& \int_{\mathbb{R}^{2}} g_{1} \varphi_{-}-2 b_{2} \int_{\mathbb{R}^{2}}\left(\mathrm{e}^{-c_{0}|z|^{2}+w_{2}}-2 \rho w_{1}\right) \varphi_{-}^{2}=0 .
\end{aligned}
$$

Recalling (2.6), we can use part (iii) of Lemma 2.1 to obtain:

$$
\int_{\mathbb{R}^{2}}\left(\mathrm{e}^{-c_{0}|z|^{2}+w_{2}}-2 \rho w_{1}\right) \varphi_{ \pm}^{2} \mathrm{~d} x=\pi \int_{0}^{+\infty}\left(\mathrm{e}^{-c_{0} r^{2}}\left(1+r^{2(N+1)}\right)-2 \rho(r) w_{1}(r)\right) \frac{r^{2(N+1)}}{\left(1+r^{2(N+1)}\right)^{2}} r \mathrm{~d} r=\frac{\pi}{2 c_{0}}
$$

Consequently, we may choose:

$$
b_{1}=\frac{c_{0}}{\pi} \int_{\mathbb{R}^{2}} g_{1} \varphi_{+}, \quad b_{2}=\frac{c_{0}}{\pi} \int_{\mathbb{R}^{2}} g_{1} \varphi_{-},
$$


and obtain that the right-hand side of (2.19) belongs to the image of the operator $L=\Delta+\rho$ when defined over $Y_{\alpha}$ (see (2.10)). Thus we derive $\psi_{1}$ and the desired conclusion follows. Next, to determine Ker $A$, take $f_{1}=f_{2}=0$ in the above computations. We find immediately, that $d_{1}=b_{1}=0, d_{2}=b_{2}=0$, and $\Delta \phi_{2}=0$. Hence, by Proposition 1.1(b), $\phi_{2}$ must be a constant. If $\phi_{2}=0$, then $g_{1}=0$ and we must take $\psi_{1} \in \operatorname{ker} L$. If $\phi_{2} \neq 0$, say $\phi_{2}=1$, then $g_{1}=-\mathrm{e}^{-c_{0}|z|^{2}+\omega_{2}}$ and $\psi_{1}$ must satisfy: $\Delta \psi_{1}+\rho \psi_{1}+\mathrm{e}^{-c_{0}|z|^{2}+\omega_{2}}=0$. Thus, $\psi_{1} \in \omega_{1}+\operatorname{ker} L$ and we conclude that $\operatorname{ker} A=W \times\{\mathbf{0}\}$ with $W \subset Y_{\alpha}^{2}$ given by

$$
W=\operatorname{Span}\left\{\operatorname{ker} L \times\{0\},\left(\omega_{1}, 1\right)\right\}
$$

as claimed.

Denote by $V_{\alpha} \subset Y_{\alpha}{ }^{2}$ the space orthogonal to $W$ defined in (2.22) with respect to the scalar product in $Y_{\alpha}{ }^{2}$. Hence, we may write:

$$
Y_{\alpha}^{2}=V_{\alpha} \oplus W
$$

Furthermore, for given $r>0$ denote by:

$$
Q_{r}=\left\{(\varepsilon, \boldsymbol{\alpha}) \in \mathbb{R}^{5}: \varepsilon \in(-r, r) \text { and } \boldsymbol{\alpha}=\left(s, \alpha_{0}, \alpha_{1}, \alpha_{2}\right) \in \mathbb{R}^{4},|\boldsymbol{\alpha}|<r\right\} .
$$

By a direct application of the Implicit Function Theorem (see Theorem 2.7.5 in [11]) we may conclude:

Theorem 2.2. Let $\alpha \in(0,1 / 2)$ there exists $\varepsilon_{0}>0$ sufficiently small and continuous functions

$$
a(\varepsilon, \boldsymbol{\alpha}): Q_{\varepsilon_{0}} \rightarrow \mathbb{C}, \quad v(\varepsilon, \boldsymbol{\alpha})=\left(\left(v_{1}(\varepsilon, \boldsymbol{\alpha})\right), v_{2}(\varepsilon, \boldsymbol{\alpha})\right): Q_{\varepsilon_{0}} \rightarrow V_{\alpha}
$$

all vanishing at $\varepsilon=0$ and $\boldsymbol{\alpha}=\mathbf{0}$, such that $\forall \varepsilon \in\left(-\varepsilon_{0}, \varepsilon_{0}\right)$ problem $\left(\mathrm{P}_{\varepsilon}\right)$ admits a four-parameterfamily of solutions $\left(u_{1, \boldsymbol{\alpha}}^{\varepsilon}, u_{2, \boldsymbol{\alpha}}^{\varepsilon}\right), \boldsymbol{\alpha}=\left(s, \alpha_{0}, \alpha_{1}, \alpha_{2}\right) \in \mathbb{R}^{4},|\boldsymbol{\alpha}|<\varepsilon_{0}$, which decompose as follows:

$$
\begin{aligned}
& u_{1, \boldsymbol{\alpha}}^{\varepsilon}=\ln \eta_{\varepsilon, a(\varepsilon, \boldsymbol{\alpha})}+\varepsilon\left((1+s) w_{1}+\alpha_{0} \varphi_{0}+\alpha_{1} \varphi_{+}+\alpha_{2} \varphi_{-}+v_{1}(\varepsilon, \boldsymbol{\alpha})\right)-2 \ln 2 g, \\
& u_{2, \boldsymbol{\alpha}}^{\varepsilon}=-c_{0}|z|^{2}+w_{2}(z)+\ln \varepsilon-2 \ln g+s+v_{2}(\varepsilon, \boldsymbol{\alpha}) .
\end{aligned}
$$

At this point, if we take $z_{1}=z_{2}=\cdots=z_{m}=0$, then problem $(\mathrm{P})$ and $\left(\mathrm{P}_{\varepsilon}\right)$ are one and the same, and in view of (2.2), (2.4), (2.6), Lemma 2.1(ii), and Proposition 1.1(c), we easily derive Theorem 2.1 .

Final remark. It would be interesting to know whether or not a result similar to Theorem 2.1 remains valid even when the vortex points do not coincide.

\section{Acknowledgements}

The authors wish to thank M. Nolasco for useful comments.

This research is supported partially by the grant no.2000-2-10200-002-5 from the basic research program of the KOSEF Korea, and by M.U.R.S.T. project: Variational Methods and Non Linear Differential Equation, Italy.

\section{Appendix A}

In order to establish Lemma 2.1, let us consider the operator

$$
L_{1}=\frac{\mathrm{d}^{2}}{\mathrm{~d} r^{2}}+\frac{1}{r} \frac{\mathrm{d}}{\mathrm{d} r}+\rho
$$


given by the restriction of the operator $L=\Delta+\rho$ over the radial functions. Thus, we wish to solve

$$
L_{1} w=f
$$

with $f(r)=-\mathrm{e}^{-c_{0} r^{2}+w_{2}(r)}=-\mathrm{e}^{-c_{0} r^{2}}\left(1+r^{2(N+1)}\right)$. Chae and Imanuvilov in [7] have obtained an integral representation for solutions of (A.2) in case

$$
f \in C^{1}\left(\mathbb{R}^{+}\right) \cap X_{\alpha} \quad \text { and } \quad \alpha \in\left(0, \frac{1}{2}\right) .
$$

Lemma A.1 (see Lemma 2.1 in [7]). Assume (A.3), then (A.2) admits a solution $w \in Y_{\alpha}$ given by the formula

$$
w(r)=\varphi_{0}(r)\left\{\int_{0}^{r} \frac{\phi_{f}(s)-\phi_{f}(1)}{(1-s)^{2}} \mathrm{~d} s+\frac{\phi_{f}(1) r}{1-r}\right\}
$$

with

$$
\phi_{f}(r)=\left(\varphi_{0}(r)\right)^{-2} \frac{(1-r)^{2}}{r} \int_{0}^{r} \varphi_{0}(t) t f(t) \mathrm{d} t, \quad \varphi_{0}(r)=\frac{1-r^{2(N+1)}}{1+r^{2(N+1)}},
$$

where $\phi_{f}(1)$ and $w(r)$ are extended by continuity at $r=1$.

In order to obtain the solution $w_{1}$ as claimed in Lemma 2.1, we shall use such a representation formula with $f(r)=-\mathrm{e}^{-c_{0} r^{2}}\left(1+r^{2(N+1)}\right)$. To this purpose, for given $n \in \mathbb{N}$, let

$$
I_{n}(r)=\int_{0}^{r}\left(1-t^{2 n}\right) t \mathrm{e}^{-c_{0} t^{2}} \mathrm{~d} t
$$

and notice that

$$
\phi_{f}(r)=-\left(\varphi_{0}(r)\right)^{-2} \frac{(1-r)^{2}}{r} I_{N+1}(r) .
$$

Lemma A.2. The following identity holds:

$$
I_{n}(r)=\frac{1}{2 c_{0}}\left[1-\frac{n !}{c_{0}^{n}}-\mathrm{e}^{-c_{0} r^{2}}\left(1-\sum_{k=0}^{n} \frac{r^{2(n-k)}}{c_{0}^{k}} \frac{n !}{(n-k) !}\right)\right] .
$$

Proof. We shall proceed by induction. For $n=1$,

$$
\begin{aligned}
I_{n=1}(r) & =\int_{0}^{r}\left(1-t^{2}\right) t \mathrm{e}^{-c_{0} t^{2}} \mathrm{~d} t \\
& =-\left.\frac{1}{2 c_{0}} \mathrm{e}^{-c_{0} t^{2}}\right|_{t=0} ^{t=r}+\frac{1}{2 c_{0}} \int_{0}^{r} \frac{\mathrm{d}}{\mathrm{d} t} \mathrm{e}^{-c_{0} t^{2}} t^{2} \mathrm{~d} t \\
& =\frac{1}{2 c_{0}}\left(1-\mathrm{e}^{-c_{0} r^{2}}+r^{2} \mathrm{e}^{-c_{0} r^{2}}-2 \int_{0}^{r} t \mathrm{e}^{-c_{0} t^{2}} \mathrm{~d} t\right)
\end{aligned}
$$




$$
\begin{aligned}
& =\frac{1}{2 c_{0}}\left[1-\mathrm{e}^{-c_{0} r^{2}}+r^{2} \mathrm{e}^{-c_{0} r^{2}}+\frac{1}{c_{0}}\left(\mathrm{e}^{-c_{0} r^{2}}-1\right)\right] \\
& =\frac{1}{2 c_{0}}\left[1-\frac{1}{c_{0}}-\mathrm{e}^{-c_{0} r^{2}}\left(1-r^{2}-\frac{1}{c_{0}}\right)\right],
\end{aligned}
$$

and (A.7) is established for $n=1$. Now assume that (A.7) holds for $n \geqslant 1$, and we proceed to prove it for $n+1$. To this end, notice that

$$
\begin{aligned}
I_{n+1}(r) & =\int_{0}^{r}\left(1-t^{2(n+1)}\right) t \mathrm{e}^{-c_{0} t^{2}} \mathrm{~d} t \\
& =\frac{1}{2 c_{0}}\left(1-\mathrm{e}^{-c_{0} r^{2}}+r^{2(n+1)} \mathrm{e}^{-c_{0} r^{2}}-2(n+1) \int_{0}^{r} t^{2 n} t \mathrm{e}^{-c_{0} t^{2}} \mathrm{~d} t\right) \\
& =\frac{1}{2 c_{0}}\left(1-\mathrm{e}^{-c_{0} r^{2}}+r^{2(n+1)} \mathrm{e}^{-c_{0} r^{2}}+2(n+1) I_{n}(r)-2(n+1) \int_{0}^{r} t \mathrm{e}^{-c_{0} t^{2}} \mathrm{~d} t\right) \\
& =\frac{1}{2 c_{0}}\left[1-\mathrm{e}^{-c_{0} r^{2}}+r^{2(n+1)} \mathrm{e}^{-c_{0} r^{2}}+2(n+1) I_{n}(r)+\frac{n+1}{c_{0}}\left(\mathrm{e}^{-c_{0} r^{2}}-1\right)\right] .
\end{aligned}
$$

Thus, if we apply the inductive assumption (A.7), substituting above, we find

$$
\begin{aligned}
I_{n+1}(r)= & \frac{1}{2 c_{0}}\left[1-\mathrm{e}^{-c_{0} r^{2}}+r^{2(n+1)} \mathrm{e}^{-c_{0} r^{2}}+\frac{2(n+1)}{2 c_{0}}\left(1-\frac{n !}{c_{0}^{n}}\right.\right. \\
& \left.\left.-\mathrm{e}^{-c_{0} r^{2}}\left(1-\sum_{k=0}^{n} \frac{r^{2(n-k)}}{c_{0}^{k}} \frac{n !}{(n-k) !}\right)\right)+\frac{n+1}{c_{0}} \mathrm{e}^{-c_{0} r^{2}}-\frac{n+1}{c_{0}}\right] \\
= & \frac{1}{2 c_{0}}\left[1-\frac{(n+1) !}{c_{0}^{n+1}}-\mathrm{e}^{-c_{0} r^{2}}\left(1-r^{2(n+1)}-\sum_{k=0}^{n} \frac{r^{2(n-k)}}{c_{0}^{k+1}} \frac{(n+1) !}{(n-k) !}\right)\right] \\
= & \frac{1}{2 c_{0}}\left[1-\frac{(n+1) !}{c_{0}^{n+1}}-\mathrm{e}^{-c_{0} r^{2}}\left(1-r^{2(n+1)}-\sum_{k=1}^{n+1} \frac{r^{2[(n+1)-k]}}{c_{0}^{k}} \frac{(n+1) !}{(n+1-k) !}\right)\right] \\
= & \frac{1}{2 c_{0}}\left[1-\frac{(n+1) !}{c_{0}^{n+1}}-\mathrm{e}^{-c_{0} r^{2}}\left(1-\sum_{k=0}^{n+1} \frac{r^{2[(n+1)-k]}}{c_{0}^{k}} \frac{(n+1) !}{(n+1-k) !}\right)\right]
\end{aligned}
$$

and the desired identity is established.

An immediate consequence of Lemma 3.2 gives

$$
I_{N+1}(r) \rightarrow \frac{1}{2 c_{0}}\left(1-\frac{(N+1) !}{c_{0}^{N+1}}\right):=\gamma_{0} \quad \text { as } r \rightarrow+\infty .
$$

Furthermore, for $r>2$, inserting (A.7) into (A.4), we find

$$
w_{1}(r)=-\varphi_{0}(r) \int_{2}^{r}\left(\frac{1+t^{2(N+1)}}{1-t^{2(N+1)}}\right)^{2} \frac{I_{N+1}(t)}{t} \mathrm{~d} t+\mathrm{O}(1)
$$




$$
\begin{aligned}
& =-\varphi_{0}(r) \int_{2}^{r}\left(\frac{1+t^{2(N+1)}}{1-t^{2(N+1)}}\right)^{2} \frac{\gamma_{0}}{t} \mathrm{~d} t+\mathrm{O}(1) \\
& =-\gamma_{0} \varphi_{0}(r) \ln r+\mathrm{O}(1) .
\end{aligned}
$$

Consequently,

$$
w_{1}(r)=\gamma_{0} \ln r+\mathrm{O}(1), \quad \text { as } r \rightarrow+\infty .
$$

On the other hand, for $r \rightarrow+\infty$,

$$
\begin{aligned}
\frac{r \mathrm{~d} w_{1}}{\mathrm{~d} r}(r) & =-r \varphi_{0}^{\prime}(r)\left(\varphi_{0}(r)\right)^{-1} w_{1}(r)-\left(\varphi_{0}(r)\right)^{-1} I_{N+1}(r)+\mathrm{O}\left(\frac{1}{r}\right) \\
& =-\left(\varphi_{0}(r)\right)^{-1}\left[I_{N+1}(r)+\frac{4(N+1) r^{2(N+1)}}{\left(1+r^{2(N+1)}\right)^{2}} w_{1}(r)+\mathrm{O}\left(\frac{1}{r}\right)\right] .
\end{aligned}
$$

So, taking into account (A.9), we immediately conclude that

$$
\frac{r \mathrm{~d} w_{1}}{\mathrm{~d} r}(r) \rightarrow \gamma_{0} \quad \text { as } r \rightarrow+\infty
$$

and, part (ii) of Lemma 2.1 follows by (3.9) and (3.10). In order to establish (iii), notice that, by direct computation, we find

$$
\frac{1}{2} L_{1}\left(\frac{1}{\left(1+r^{2(N+1)}\right)^{2}}\right)=\frac{8(N+1)^{2} r^{4 N+2}}{\left(1+r^{2(N+1)}\right)^{4}}=\frac{\rho(r) r^{2(N+1)}}{\left(1+r^{2(N+1)}\right)^{2}}
$$

while, by definition, $L_{1} w_{1}=-\mathrm{e}^{-c_{0} r^{2}}\left(1+r^{2(N+1)}\right)$. Therefore, in view of the asymptotic behaviors (A.9) and (A.10) of $w_{1}$ as $r \rightarrow+\infty$, we can use integration by parts, to obtain:

$$
\begin{aligned}
& \int_{0}^{+\infty} \rho(r) \frac{r^{2(N+1)}}{\left(1+r^{2(N+1)}\right)^{2}} w_{1}(r) r \mathrm{~d} r \\
& =\frac{1}{2} \int_{0}^{+\infty} L_{1}\left(\frac{1}{\left(1+r^{2(N+1)}\right)^{2}}\right) w_{1}(r) r \mathrm{~d} r \\
& =\frac{1}{2} \int_{0}^{+\infty} \frac{\mathrm{d}}{\mathrm{d} r}\left(r \frac{\mathrm{d}}{\mathrm{d} r} \frac{1}{\left(1+r^{2(N+1)}\right)^{2}}\right) w_{1}(r) \mathrm{d} r+\frac{1}{2} \int_{0}^{+\infty} \rho(r) w_{1}(r) \frac{1}{\left(1+r^{2(N+1)}\right)^{2}} r \mathrm{~d} r \\
& =\frac{1}{2} \int_{0}^{+\infty} \frac{1}{\mathrm{~d} r}\left(r \frac{\mathrm{d}}{\mathrm{d} r} w_{1}(r)\right) \frac{1}{\left(1+r^{2(N+1)}\right)^{2}} \mathrm{~d} r+\frac{1}{2} \int_{0}^{+\infty} \rho(r) w_{1}(r) \frac{1}{\left(1+r^{2(N+1)}\right)^{2}} \mathrm{~d} r \\
& =\frac{1}{2} \int_{0}^{+\infty} L_{1} w_{1} \frac{1}{\left(1+r^{2(N+1)}\right)^{2}} r \mathrm{~d} r=-\frac{1}{2} \int_{0}^{+\infty} \frac{\mathrm{e}^{-c_{0} r^{2}}}{1+r^{2(N+1)}} r \mathrm{~d} r .
\end{aligned}
$$

Consequently,

$$
\int_{0}^{+\infty}\left(\frac{\mathrm{e}^{-c_{0} r^{2}} r^{2(N+1)}}{1+r^{2(N+1)}}-\frac{2 \rho(r) w_{1}(r) r^{2(N+1)}}{\left(1+r^{2(N+1)}\right)^{2}}\right) r \mathrm{~d} r=\int_{0}^{+\infty} \mathrm{e}^{-c_{0} r^{2}} r \mathrm{~d} r=\frac{1}{2 c_{0}} .
$$




\section{References}

[1] A.A. Abrikosov, On the magnetic properties of superconductors of second group, Sov. Phys. JETP 5 (1957) 1174-1182.

[2] J. Ambjorn, P. Olesen, A magnetic condensate solution of the classical electroweak theory, Phys. Lett. B 218 (1989) 67-71.

[3] J. Ambjorn, P. Olesen, On electroweak magnetis, Nucl. Phys. B 315 (1989) 606-614.

[4] J. Ambjorn, P. Olesen, A condensate solution of the electroweak theory which interpolates between the broken and symmetry phase, Nucl. Phys. B 330 (1990) 193-204.

[5] D. Bartolucci, G. Tarantello, The Liouville equations with singular data and their applications to electroweak vortices, Comm. Math. Phys. 229 (2002) 3-47.

[6] H. Brezis, F. Merle, Uniform estimates and blow-up behaviour for solutions of $-\Delta u=V(x) \mathrm{e}^{u}$ in two dimensions, Comm. Partial Differential Equations 16, (8,9), 1223-1253.

[7] D. Chae, O.Yu. Imanuvilov, The existence of non-topological multivortex solutions in the relativistic self-dual Chern-Simons theory, Comm. Math. Phys. 215 (2000) 119-142.

[8] W. Chen, C. Li, Qualitative properties of solutions to some nonlinear elliptic equations in $\mathbb{R}^{2}$, Duke Math. J. 71 (2) (1993) $427-439$.

[9] G. 't Hooft, A property of electric and magnetic flux in nonabelian gauge theories, Nucl. Phys. B 153 (1979) 141-160.

[10] C.H. Lai (Ed.), Selected Papers on Gauge Theory of Weak and Electromagnetic Interactions, World Scientific, Singapore.

[11] L. Nirenberg, Topics in Nonlinear Analysis, in: Courant Lecture Notes in Math., American Mathematical Society, 2001.

[12] J. Prajapat, G. Tarantello, On a class of elliptic problems in $\mathbb{R}^{2}$ : symmetry and uniqueness results, Proc. Royal Soc. Edinburgh 131 (4) (2001) 967-985.

[13] J. Spruck, Y. Yang, On multivortices in the electroweak theory I: existence of periodic solutions, Comm. Math. Phys. 144 (1992) 1-16.

[14] J. Spruck, Y. Yang, On multivortices in the electroweak theory II: existence of Bogomol'nyi solutions in $\mathbb{R}^{2}$, Comm. Math. Phys. 144 (1992) 215-234

[15] C.H. Taubes, Arbitrary $N$-vortex solutions to the first order Ginzburg-Landau equation, Comm. Math. Phys. 72 (1980) $277-292$.

[16] C.H. Taubes, On the equivalence of first order and second order equations for gauge theories, Comm. Math. Phys. 75 (1980) $207-227$.

[17] Y. Yang, Solitons in Field Theory and Nonlinear Analysis, in: Springer Monographs in Math., Springer-Verlag, New York, 2001. 